\title{
Antiretroviral Therapy in the Malawi Police Force: Access to Therapy and Treatment Outcomes
}

Simon D. Makombe ${ }^{1}$ Andreas Jahn ${ }^{2,3}$, Hannock Tweya ${ }^{2}$, Stuart Chuka ${ }^{4}$, Joseph Kwong-Leung Yu $^{5}$, Bethany Hedt ${ }^{6}$, Ralf Weigel ${ }^{2}$, Amon Nkhata ${ }^{1}$, Erik J. Schouten ${ }^{1,7}$, Kelita Kamoto ${ }^{1}$, Anthony D. Harries ${ }^{1,8,9}$,

\footnotetext{
1 HIV Unit, Ministry of Health, PO Box 30377, Lilongwe, Malawi

2 Lighthouse Trust, Lilongwe, Malawi

3 International Training and Education Center on HIV, Seattle, USA

4 Malawi Business Coalition against AIDS, Blantyre, Malawi

5 Taiwan Medical Mission, Mzuzu Central Hospital, Mzuzu, Malawi

6 Harvard School of Public Health, USA

7 Management Sciences for Health, Lilongwe, Malawi

8 Family Health International, Malawi Country Office, Lilongwe, Malawi

9 London School of Hygiene and Tropical Medicine, Keppel Street, London, UK
}

Correspondence: Professor AD Harries, Family Health International, Malawi Country Office, Arwa House, 3rd Floor, PO Box 30455,

Lilongwe 3, Malawi

Fax: + 2651774307

Email: adharries@malawi.net

\begin{abstract}
A national survey was carried out in all the 103 public sector and 38 private sector facilities in Malawi providing antiretroviral therapy (ART) to determine uptake of ART and subsequent treatment outcomes in police force personnel. All patients registered for ART and their subsequent treatment outcomes were censored on December 31st 2006. There were 85168 patients started on ART in both public and private sectors, of whom $463(0.6 \%)$ were police force personnel. Of police force personnel starting ART, 17\% were in WHO clinical stage 1 or 2 with a CD4-lymphocyte count of $\leq 250$ cells / $\mu \mathrm{L}$ and $83 \%$ were in stage 3 or 4 . Treatment outcomes of police force personnel by the end of December 2006 were $302(65 \%)$ alive and on ART at their registration facility, 59 $(13 \%)$ dead, $30(7 \%)$ lost to follow-up, 1 stopped treatment and $71(15 \%)$ transferred to another facility. Their probability of being alive on ART at 6-, 12- and 18-months was $83.2 \%$, $78.6 \%$ and $76.7 \%$ respectively. There has been a good access of police force personnel to ART since national scale up commenced with good treatment outcomes, and this should serve as an example for other police forces in the region.
\end{abstract}

\section{Introduction}

Malawi, a land-locked country in central-southern Africa, has a population of between 11.5 to 12.5 million and is among the poorest countries in the world. Since the beginning of the 1990 s, the country has been affected by a severe generalised HIV-epidemic and 14\% of adults aged 15-49 years are estimated to be HIV-positive ${ }^{1}$. Malawi's economy and public services are further weakened by long-term morbidity among people in their most productive age and by an estimated 80000 premature deaths due to AIDS each year ${ }^{1}$. A survey on human resources in the public sector between 1990 and 2000 showed that the Government was spending large sums of scarce resources on ever-increasing numbers of sick officers and funerals, and this diversion of resources was heavily affecting the core business of Government ${ }^{2,3}$. During this 10 -year period, the Malawi Police Force, with an average national complement of 6000 employed staff, experienced an annual attrition rate of $6 \% 0^{2}$. Nearly half of this attrition was due to death, the majority of deaths occurring in male police officers between the ages of 30-39 years. Although the causes of death were difficult to ascertain in the UNDP survey, many deaths were thought to be attributable to HIV/ AIDS. The Police Force is likely to be particularly susceptible to the risk of HIV infection due to high professional mobility and the opportunities that thereby take place for acquisition of infection ${ }^{4}$.

Since 2004, Malawi has been scaling up antiretroviral therapy $(\mathrm{ART})^{5}$, and treatment is available free of charge in the public sector from hospitals, health centres and clinics, and can be obtained at a subsidised rate from the private sector as well. One of Malawi's police hospitals in the Southern Region of the country was included in the first round of the scale up and in June 2005, a second police clinic in the Central Region was also included as an ART site. The police force and their families and the surrounding catchment population can attend any one of the 351 static HIV testing sites in the country for free HIV testing and counselling: testing and counselling is voluntary, although in health facilities it is often provider-initiated in sick patients as this may facilitate diagnosis and management ${ }^{6}$. There is no mandatory HIV testing. Persons who are found to be HIV-positive are usually referred to facilities with ART clinics for assessment about eligibility for ART. Police force personnel can access free ART from either of the two special police clinics, and are also able to receive ART drugs from any public or private sector site in the country.

Data on all ART patients in both public and private sectors are recorded using a standardised system for monitoring case finding and treatment outcomes ${ }^{7}$, and this includes information on occupation and demographic status. Every three months the HIV Unit of the Ministry of Health and its partners conduct rigorous supervision at all ART sites ${ }^{8}$, and the data can be considered nationally complete and reliable. However, the data is not disaggregated at the national level, but information on occupation allows the opportunity to examine uptake, retention in therapy and survival for subgroups of the population.

The Malawi Police Force has a current complement in 2007 of about 11000 police officers, and has the responsibility of maintaining law and order in the country by providing internal security services of magnitude and quality necessary for peace and protection of the people and their property. While there are no data on HIV prevalence in the police force or on number of staff in need of ART, based on a national adult HIV-prevalence rate of 14\%, about 1540 police officers could be expected to be HIV-infected, of whom about $25 \%$ $-30 \%$ or 400 police officers might be in immediate need of ART. Given the importance of maintaining healthy personnel in the country's police force, we were interested to know how many police staff had ever accessed ART and how many were retained alive on therapy. Using routinely collected data from Malawi's national monitoring and evaluation system for 
ART, we therefore conducted an audit of personnel from the Malawi Police Force in the country to look at these issues.

\section{Methods \\ Background}

From June 2004 onwards, ART was made free as part of national policy within the public sector and national roll-out started with standardised monitoring systems established in every ART site. The details have been previously reported, and are only briefly described below. A simple, standardised approach is used which focuses on:- use of one generic, fixed dose combination treatment (stavudine, lamivudine, nevirapine); a standardised system of registration, monitoring and reporting of cases and outcomes; and quarterly monitoring and supervision visits to all ART sites. Patients are eligible for ART if they have a positive HIV test, they or their guardians understand the implications of therapy, and they are assessed in WHO Clinical Stage 3 or 4 or have a CD4-lymphocyte count $<250$ cells $/ \mu \mathrm{L}$ for adults ${ }^{9,10}$. Because of a shortage of CD4-count measuring capacity in the country, most patients are started on ART based on clinical criteria only. Patients are seen at two weeks after ART initiation and then routinely every month for clinical assessment and ART-dispensing. In most ART clinics, there is no capacity for measuring CD4lymphocyte counts or viral load, and follow-up assessment is based solely on clinical reviews. The monthly visit interval is extended to 2 months if patients have completed 6 months of ART without complications and have been shown on pill counting to be adherent to therapy. If patients are sick or pill counts show poor medication adherence, the monthly visits are maintained unchanged. Patients with drug side effects are changed to alternative ART regimens, the main changes being the substitution of stavudine to zidovudine and nevirapine to efavirenz. If there is suspicion of clinical failure on first line $\mathrm{ART}^{10}$, the patients are referred to one of the central hospitals in the country, where CD4-lymphocyte counts and sometimes viral load are performed to determine if there is a need to switch to a second line regimen.

Malawi's national monitoring system for ART uses one patient master card for each patient and one ART register per facility ${ }^{7,8}$. At enrolment, patient demographics, occupation, stage defining conditions and clinical stage are recorded on the master card and copied into the register. At every ART visit, follow-up details with their dates are entered in the master card, and these include transfer to another ART clinic, treatment discontinuation and death. Patients who fail to return for 3 months are marked as 'lost to follow-up'. Although active follow-up of patients who fail to return to clinic has not been made mandatory due to resource constraints, more than half of the facilities in the national programme are consistently attempting to trace these patients through community visits. A patient cohort analysis is conducted at all sites every quarter. In preparation for this, clinic staff systematically review the follow-up status of all patients, updating the master cards and register with the latest outcomes.

The HIV Unit of the Ministry of Health and its partners conduct quarterly supervision and monitoring visits to all ART sites in the country. The supervisors check the accuracy, completeness and consistency of the register and master cards, and update incorrect records during the site visit.
Cohort analyses are checked, corrected for any mistakes and collected for aggregation and national level reporting.

A similar system is used in the private sector, with the same monitoring tools and same quarterly supervision conducted by a clinical officer employed by the Malawi Business Coalition against AIDS. The only difference is that patients have to pay the equivalent of MK 500 per month for their ART, and this payment is similar regardless of the type of regimen.

\section{Data Collection and Analysis}

The survey on access and retention of police staff on ART was conducted during supervision and monitoring visits to all 103 public sector ART clinics and 38 private sector clinics, which took place between January and March 2007. In addition to the routine collection of data, all ART clinic registers were screened for police staff who had accessed ART up to December 31st 2006. For all police staff identified, the following data were transcribed onto a structured form: sitespecific registration number; sex; date, age and WHO clinical stage at ART initiation; pulmonary or extra-pulmonary TB as a stage-defining condition; date and type of follow-up outcome. The data were checked, entered and cleaned in MS Access and analysed using STATA 9.2. Observations were censored if patients were alive and on ART by 31st December 2006. Adverse outcomes of death, lost to followup and ART discontinuation were regarded as 'failure events' and their dates were recorded, while transfers to other ART clinics were regarded as censoring events and their dates were also recorded. Categorical variables between police staff and patients who were not police staff were analysed and compared using the chi squared test with odds ratios (OR). The probability of survival on ART was estimated using the Kaplan-Meier method. The level of significance for all comparisons was set at $\mathrm{P}=0.05$ or less, and $95 \%$ confidence intervals (CI) were used throughout.

\section{Results}

\section{Total National Patient Cohort}

By December 31st 2006, a total of 85168 patients had registered for ART in Malawi: 81821 (96\%) at the 103 public sector and $3347(4 \%)$ at the 38 private sector clinics. There were $17(16.5 \%)$ public sector ART sites with a capacity to perform CD4-lymphocyte counts. Of patients started on ART, 51797 (60.8\%) were female and 79259 (93.1\%) were aged 15 years or above. There were 10976 (12.9\%) patients who had accessed ART in WHO clinical stage 1 or 2 with a CD 4 count $\leq 250$ cells $/ \mu \mathrm{L}, 54477(64.0 \%)$ in stage 3 and $19715(23.1 \%)$ in stage 4. By the end of December 2006, $59980(70.4 \%)$ patients were alive and on ART, $7382(8.7 \%)$ had transferred to another clinic, $372(0.4 \%)$ had stopped ART, $7885(9.3 \%)$ were lost to follow-up and 9549 (11.2\%) had died. The routine cohort treatment outcome analyses that are performed every three months in the public sector showed that $74 \%$ of 10608 patients registered for ART between April and June 2006, and 61\% of 7871 patients registered for ART between October and December 2005 were alive and on ART at 6 and 12 months after enrolment, respectively. 
Table 1: Demographic, clinical features and treatment outcomes censored on December 31 st 2006 in police staff and non-police staff starting free $A R T$ in the public sector in Malawi

\begin{tabular}{|l|l|l|l|}
\hline & Police staff & Non-police staff & $\begin{array}{l}\text { Odds ratio } \\
(95 \% \mathrm{Cl})^{*}\end{array}$ \\
\hline Number & 463 & 81358 & \\
\hline Males & $374(75.0 \%)$ & $31285(38.5 \%)$ & $6.7[5.3-8.5]^{*}$ \\
\hline $\begin{array}{l}\text { Known reason } \\
\text { for starting ART }\end{array}$ & 456 & 81358 & \\
\hline $\begin{array}{l}\text { WHO Stage 1 } \\
\text { and 2 }\end{array}$ & $76(16.6 \%)$ & $9757(12.0 \%)$ & $1.5[1.1-1.9]^{*}$ \\
\hline WHO Stage 3 & $245(53.7 \%)$ & $52778(64.9 \%)$ & $0.6[0.5-0.8]^{*}$ \\
\hline WHO Stage 4 & $135(29.6 \%)$ & $18823(23.1 \%)$ & $1.4[1.1-1.7]^{*}$ \\
\hline $\begin{array}{l}\text { Pulmonary } \\
\text { Tuberculosis }\end{array}$ & $55(12.1 \%)$ & $11312(13.9 \%)$ & $0.8[0.6-1.1]$ \\
\hline $\begin{array}{l}\text { Extra-pulmonary } \\
\text { tuberculosis }\end{array}$ & $8(1.8 \%)$ & $1800(2.2 \%)$ & $0.8[0.4-1.6]$ \\
\hline $\begin{array}{l}\text { Treatment } \\
\text { outcomes: }\end{array}$ & & & $1.9[1.5-2.5]^{*}$ \\
\hline $\begin{array}{l}\text { Alive on ART at } \\
\text { the clinic }\end{array}$ & $302(65.2 \%)$ & $57054(70.1 \%)$ & $0.8[0.7-0.9]^{*}$ \\
\hline $\begin{array}{l}\text { Transferred out } \\
\text { to another clinic }\end{array}$ & $71(15.3 \%)$ & $6949(8.5 \%)$ & $1.9[1.90 .9-1.4]$ \\
\hline $\begin{array}{l}\text { Alive + Trans } \\
\text { ferred out }\end{array}$ & $373(80.5 \%)$ & $64003(78.7 \%)$ & $1.1[0.9-1.5]^{*}$ \\
\hline Dead & $59(12.7 \%)$ & $9268(11.4 \%)$ & $1.2[0.9-1.5]$ \\
\hline Lost to follow-up & $30(6.5 \%)$ & $7723(9.5 \%)$ & $0.7[0.4-0.9]^{*}$ \\
\hline Stopped therapy & $1(0.2 \%)$ & $364(0.5 \%)$ & $0.5[0.03-3.2]$ \\
\hline
\end{tabular}

* Odds ratios which are significant at $p<0.05$

\section{Police Staff on ART and Comparisons with Non- police Staff in the Public Sector}

Information on occupation was available for 82297 (96.6\%) patients in the national public and private sector cohort. There were 463 police staff $(0.6 \%$ of the cohort), with a mean age at ART initiation of 38 (range 23-70) years. Police staff had accessed ART from 65 public sector facilities, and this included 70 staff $(15 \%)$ who had registered at one of the two police ART clinics. There were 15 (23.1\%) clinics accessed by police staff that had capacity to perform CD4counts, which was not significantly higher than for the general population [OR 1.5, 95\% CI 0.6-3.5]. No police force staff had registered for ART in the private sector. Forty five $(10 \%)$ police staff had accessed ART before the start of the national scale-up programme when treatment generally had to be paid for.

In the public sector sites, demographic, clinical features and outcomes by the end of December 2006 in police staff and the other non-police staff patients are shown in Table 1.

In the police force, there were more males accessing ART, more patients started on ART due to being in WHO Stage 1 and 2 with a low CD 4 count, but in those registered through clinical staging more were in WHO Stage 4 compared with non-police staff. Similar proportions of patients in each group were started on ART due to tuberculosis. There were $26(6 \%)$ police staff started on ART due to Kaposi's Sarcoma: no data on Kaposi's Sarcoma were available for the non-police staff.

The median observation time for the whole police staff cohort was 7.9 months (range 0.1 to 54 months), and the entire cohort had accumulated 409 person-years of observation. In the police force, fewer patients were alive and retained at their original registration sites, more had been transferred out to another clinic and fewer had been lost to follow-up compared with non-police staff (Table 1). However, when patients alive and on ART were combined with those who had transferred out, there were no differences between the two groups. The number of police staff accessing ART and the number retained alive and on ART by calendar period are shown in Figure 1. Their probability of being alive and on ART 6,12 and 18 months after treatment registration was $83.2 \%, 78.6 \%$, and $76.7 \%$ respectively (see Figure 2 ).

\section{Discussion}

We believe that this is the first national survey in SubSaharan Africa of police staff on ART examining uptake and retention on therapy. By the end of 2006 the nationwide coverage with ART clinics had enabled a total of 463 police staff throughout the country to access ART. Based on the estimated number thought to be in immediate need of ART, coverage in the Malawian police force is reasonably good. The fact that the majority of police force patients on ART were registered at regular public ART clinics and that only $15 \%$ were managed by the police ART facilities is an indication that the wide geographic availability of ART services is crucial for this sector, and that police staff have been able to access ART in the vicinity of their posting.

Police staff on ART differed in demographic and clinical characteristics from other patients accessing ART from public sector sites on two main counts:- there were more males and more patients started on ART due to being in Stage $1 \& 2$ with a low CD4 count. These differences are due firstly to the majority of police staff in Malawi being male. Secondly, although the number of ART clinics with capacity to perform CD4 counts was not significantly different between police officers and the general population, the two main police clinics are situated in cities where in fact there is relatively good access to laboratories that can perform these tests. In terms of treatment outcomes, there was a lower loss to follow-up and a higher transfer-out rate compared with non-police patients. Follow-up among police staff on ART, through institutional arrangements, is likely to be better than in the general ART patient cohort, hence the low default rate. However, professional mobility among police officers is high due to changing postings to different parts of the county and this is reflected in the high proportion of clinic transfers. Recent operation research (Yu, unpublished observations) shows that the majority of patients who transfer-out do in fact transfer-in at another site with the majority of these patients staying alive and on therapy. Survival probabilities in the police force up to 12-18 months were generally good and compare favourably with similar cohort studies conducted elsewhere in Malawi ${ }^{11,12}$ and in other African countries ${ }^{13,14,15,16}$. Although police force staff on ART had better survival outcomes compared with the national cohort, direct comparisons are difficult because of different methods of measuring survival data (individual survival data in the case of the police force compared with group cohort survival in the general patient cohort). 


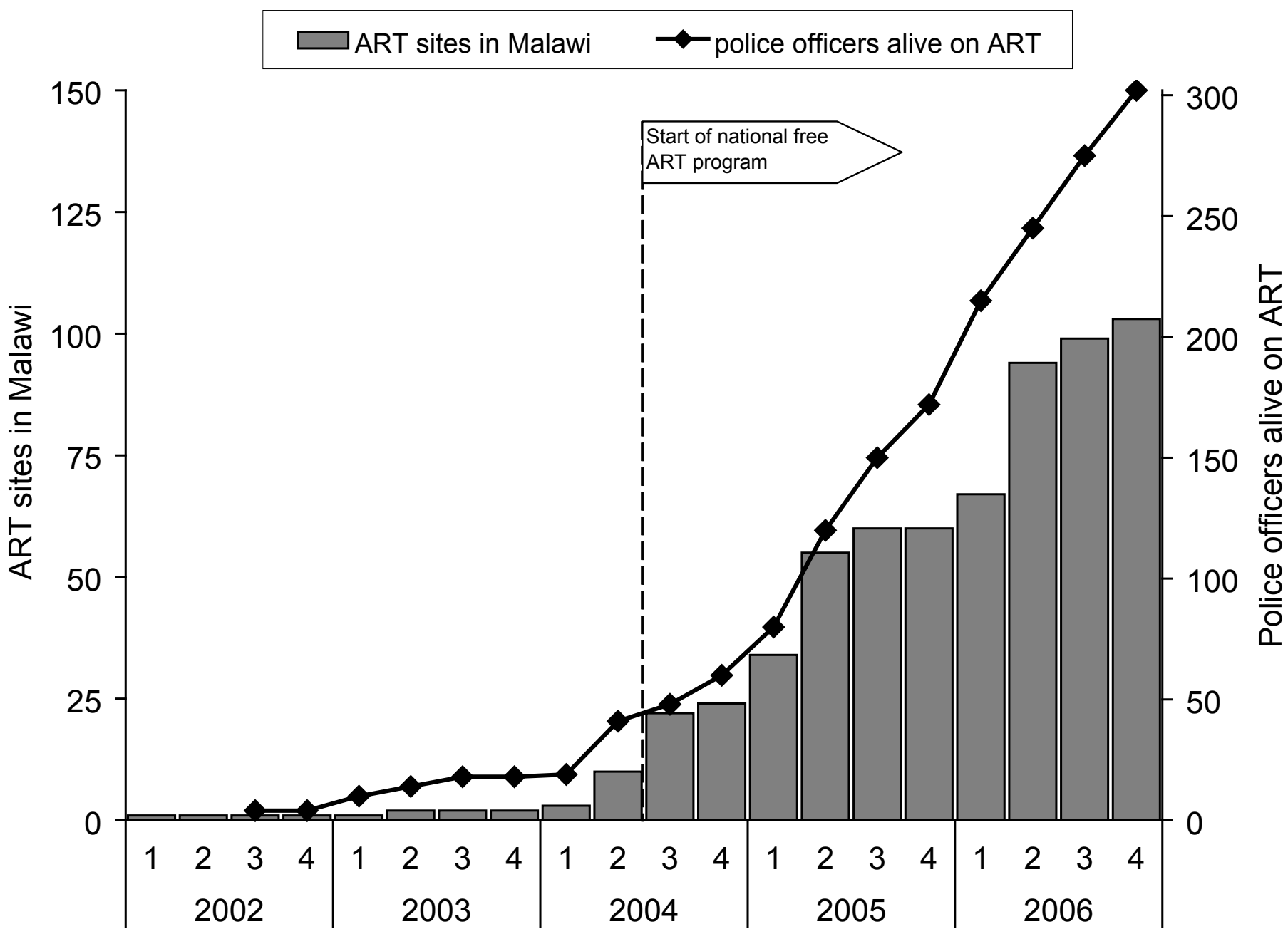

Good survival probabilities amongst police staff on ART are probably due to the higher proportion of police staff accessing ART at an earlier stage of HIV-disease. Without ART, HIV-infected patients assessed in WHO Clinical Stage 3 or 4 have a very poor prognosis in Malawi ${ }^{17}$, while on ART the prognosis is transformed ${ }^{5,11}$.

This operational study was based on data from the routine national monitoring system, and has all the limitations of this type of research. Occupation was unknown for about $4 \%$ of patients, and this may have lead to a small undercounting of the number of police force personnel who accessed ART in the country. It is also possible that some patients felt uncomfortable registering as a police officer at an ART clinic, and may have given a different occupation to ART clinic staff. The average observation time of the national cohort of police staff is still relatively short, and longer term outcomes could not be reliably assessed. Finally, in comparing police staff with non-police staff, the latter includes a small proportion of children who should not really be compared with adult police officers. Unfortunately, it is not possible in the routine data collection exercises to disaggregate outcome data for adults or children. The strengths of the study are that this is a full national survey, all ART-providing facilities in the public and private sector of Malawi were included, and the analysis is likely to provide near-complete information on all police force personnel who have accessed treatment in the country, provided that they have been accurately recorded in the occupation column of the register. Malawi has established a standard, national monitoring system for ART used by all public and private sector facilities, the data are routinely validated during quarterly supervision through cross-checking of master cards and ART registers, and this should result in relatively high data quality.

The Malawi Police Force has supported the roll-out of ART in Malawi and made available free ART to its personnel and the general public at the two police clinics. There was only a small number of police staff who had accessed ART before the start of the national ART programme, at a time when clinics were usually charging for this service. By the end of 2006, there were free public ART clinics in all districts in Malawi and increasing numbers of police staff are surviving on ART. The survey conducted from 1990 - 20002 should be now repeated in order to re-examine attrition and in particular mortality within the police sector so that the impact of ART in Malawi can be better quantified within sub-sections of the population.

\section{Acknowledgements}

We thank Dr Anne Ben-Smith for her help with processing and submitting the paper online.

\section{References}

1. National AIDS Commission. HIV \& syphilis sero-survey and national HIV prevalence estimates report. 2005. Lilongwe, Malawi: 


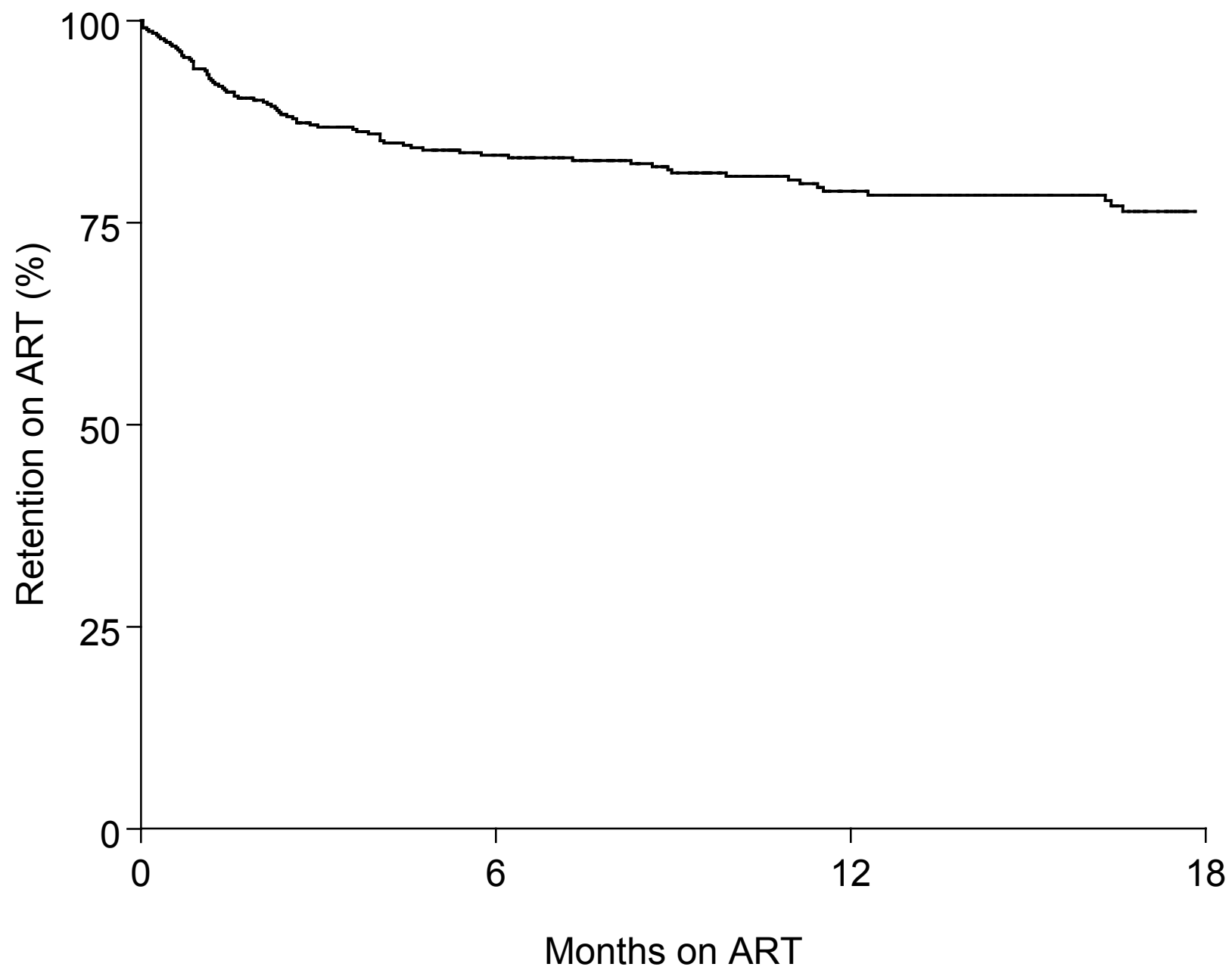

National AIDS Commission.

2. UNDP. The impact of HIV/AIDS on human resources in the Malawi Public Sector. Report, February, 2002.

3. Matewele ER. The impact of HIV/AIDS on human resources in the Malawi public sector.15th International AIDS Conference, International AIDS Society, Bangkok, Thailand. 2004, July 11-16, 15: abstract no. TuPeE5352.

4. Piot P, Greener R, Russell S. Squaring the circle: AIDS, poverty, and human development. PloS Medicine 2007; 4: e314.

5. Harries AD, Libamba E, Schouten EJ. Scaling up antiretroviral treatment in resource-poor settings. Lancet 2006; 367: $1870-$ 1872.

6. National AIDS Commission. National HIV/AIDS policy. A call to renewed action. 2003. ISBN No: 99908-73-07-0. Office of the President and Cabinet.

7. Libamba E, Makombe S, Harries AD, et al. Scaling up antiretroviral therapy in Africa: learning from tuberculosis control programmes - the case of Malawi. Int J Tuberc Lung Dis 2005; 9:1062 - 1071.

8. Libamba E, Makombe S, Mhango E, et al. Supervision, monitoring and evaluation of nationwide scale up of antiretroviral therapy in Malawi. Bull World Health Organ 2006; 84: 320 - 326.

9. WHO. Antiretroviral therapy of HIV infection in adults and adolescents in resource-limited settings, towards universal access: recommendations for a public health approach. 2006. Geneva, World Health Organization.

10. Ministry of Health, Malawi.Treatment of AIDS. Guidelines for the use of antiretroviral therapy in Malawi. Second edition. April 2006,
Lilongwe, Malawi.

11. Ferradini L, Jeannin A, Pinoges L, et al. Scaling up of highly active antiretroviral therapy in a rural district of Malawi: an effectiveness assessment. Lancet 2006; 367: 1335- 1342.

12. Makombe SD, Jahn A, Tweya $\mathrm{H}$, et al. A national survey of teachers on antiretroviral therapy in Malawi: access, retention in therapy and survival. PLoS One 2007; issue 7: e620.

13. Wester CW, Kim S, Bussmann H, et al. Initial response to highly active antiretroviral therapy in HIV-1C-infected adults in a public sector treatment program in Botswana. J Acquir Immune Defic Syndr 2005; 40: $336-343$.

14.Lawn SD, Myer L, Orrell C, Bekker L-G, Wood R. Early mortality among adults accessing a community-based antiretroviral service in South Africa: implications for programme design. AIDS 2005; 19 : $2141-2148$.

15. Wools-Kaloustian K, Kimaiyo S, Diero L, et al. Viability and effectiveness of large-scale HIV treatment initiatives in sub-Saharan Africa: experience from western Kenya. AIDS 2006; 20: 41-48.

16. Calmy A, Pinoges L, Szumilin E, et al. Generic fixed-dose combination antiretroviral treatment in resource-poor settings: multicentric observational cohort. AIDS 2006; 20: 1163 - 1169.

17. van Oosterhout JG, Laufer MK, Graham SM, et al. A communitybased study on the incidence of trimethoprim-sulfamethoxazolepreventable infections in Malawian adults living with HIV. J Acquir Immune Defic Syndr 2005; 39: 626 - 631Clinton WJ. Turning the tide on the AIDS epidemic. N Eng J Med 2003; 348: 1800-1802. 